\title{
Topology Optimization of Metallic Antennas
}

\author{
Emadeldeen Hassan, Eddie Wadbro, and Martin Berggren
}

\begin{abstract}
We introduce an approach to carry out layout optimization of metallic antenna parts. An optimization technique first developed for the optimization of load-bearing elastic structures is adapted for the purpose of metallic antenna design. The local conductivity values in a given region are used as design variables and are iteratively updated by a gradient-based optimization algorithm. Given a set of time-domain signals from exterior sources, the design objective is here to maximize the energy received by the antenna and transmitted to a coaxial cable. The optimization proceeds through a sequence of coarsely-defined lossy designs with successively increasing details and less losses as the iterations proceed. The objective function gradient is derived based on the FDTD discretization of Maxwell's equations and is expressed in terms of field solutions of the original antenna problem and an adjoint field problem. The same FDTD code, but with different wave sources, is used for both the original antenna problem and the adjoint problem. For any number of design variables, the gradient is evaluated on the basis of only two FDTD simulations, one for the original antenna problem and another for the adjoint field problem. We demonstrate the capability of the method by optimizing the radiating patch of both UWB monopole and microstrip antennas. The UWB monopole is designed to radiate over a wide frequency band 1$10 \mathrm{GHz}$, while the microstrip patch is designed for single and dual frequency band operation. In these examples, there are more than 20,000 design variables, and the algorithm typically converges in less than 150 iterations. The optimization results show a promising use of the proposed approach as a general method for conceptual design of near-resonance metallic antennas.
\end{abstract}

Index Terms-topology optimization, finite-difference timedomain (FDTD), adjoint field problem, ultrawideband antennas (UWB), microstrip antennas, coaxial feed model.

\section{INTRODUCTION}

$\mathbf{T}$ HE current revolution in wireless systems raises the interest in the design of complex antenna structures. Moreover, applications such as microwave imaging and nondestructive evaluation and testing using electromagnetic waves benefit from the access to antennas that satisfy specific characteristics [1]. Currently, antenna design often relies on accurate numerical methods that solve Maxwell's equations, such as the method of moments (MoM), the finite element method (FEM), and the FDTD method [2].

Classical design methods start with an existing geometry that is found in the literature or inspired by prior knowledge. Then, various parameter studies are carried out to optimize certain objectives, such as the reflection coefficient, the directivity, the polarization, or the mutual coupling between antennas. Generally, the quality of the optimized designs depends on how the design domain is parameterized and the number of design variables used in the parameterization. The

Manuscript received April 3, 2013; revised January 15, 2014; accepted February 22, 2014. The authors are with the Department of Computing Science, Umeå University, SE-901 87 Umeå, Sweden (email: emad@cs.umu.se; eddie.wadbro@cs.umu.se; martin.berggren@cs.umu.se). use of a large number of design variables results in a large set of feasible solutions and increases the possibility to obtain a high-performance design. The cost of handling a large number of design variables will depend on the optimization algorithm.

Evolutionary algorithms, such as genetic algorithms and particle swarm optimization, are extensively used in the literature to optimize various electromagnetic devices [3]-[6]. Evolutionary algorithms typically make use of objective function values only, which make them simple to use as black-box software. However, the small amount of information contained in only a sampling of objective function values, together with the random strategies employed to update the design variables, make these types of algorithms inefficient for optimization problems with a large number of design variables [7].

The term topology optimization is often used to label the most general type of design optimization methods, in which the shapes as well as the connectivity of individual parts of the device are subject to design. The most common way of carrying out topology optimization is through the material distribution approach, in which the design domain is divided into small elements, which together represent an image of the device. A design variable is assigned to each element to indicate presence or absence of a material, and the various designs are represented as varying coefficients in the governing equations. The material distribution approach to topology optimization was originally developed to design load-carrying elastic structures [8], but the method has been successfully extended also to other areas of engineering, such as for the design of acoustics and optics devices [8]-[11]. Instead of optimizing directly over design variables associated with small elements, an alternative topology optimization technique relies on a representation of the geometry through level sets: the device boundary is defined as the zero-level contour of a higher-dimensional scalar function [12].

For topology optimization problems, the number of design variables can easily reach thousands and even millions for 2D and 3D design problems [13]. Gradient-based optimization techniques are generally preferred to solve such large scale problems. A main reason for this choice is that the gradient of an objective function contains a massive amount of information, and gradients can in many cases be very efficiently computed using solutions of associated adjoint field problems [14]-[20]. In the electromagnetics community, topology optimization techniques have been introduced for the design of magnetic devices by Dyck et al. [21], [22] and for the design of dielectric substrates for bandwidth improvement of patch antennas by Kiziltas et al. [23]. Further, Nomura et al. [18] proposed to use topology optimization for the design of dielectric resonator antennas to operate with enhanced bandwidth. However, to the best of our knowledge, the use of topology optimization methods for the design of metallic 
antennas has only been reported by Erentok and Sigmund [24], who used the material distribution approach, and by Zhou et al. [25], who used a method based on level sets. Both these studies used frequency domain methods for a single frequency design.

In this article, we present a version of the material distribution approach to topology optimization for the design of metallic antennas. The problem formulation is based on the 3D Maxwell's equations, discretized with the FDTD method [26]. The use of time-domain solutions of Maxwell's equations has the advantage of an effective treatment of a band of frequencies at once. The objective function is formulated as the energy received by the antenna from a set of far field sources and transmitted to a coaxial feed line; that is, the antenna is designed based on its receiving mode. A sensitivity analysis reveals that the derivative of the objective function with respect to perturbations of the conductivity distribution can be expressed in terms of the solution of an adjoint field problem. For robust performance of gradient-based optimization algorithms, the derivative used by the algorithm should accurately represent the derivative of the discrete objective function. For this reason, we have derived the adjoint equations and the expression for the gradient in the fully discrete case, based on the FDTD equations with the UPML parameters and the discrete coaxial feed model included. We use the proposed technique for a complete layout optimization of the metallic radiating elements of two antenna types, the UWB monopole antenna and the microstrip antenna. The main novelty of our contribution is that we, in contrast to standard optimization methods for antennas, can carry out large scale conceptual antenna designs with orders of magnitude less iterations than the number of design variables. To demonstrate the capability of this new approach, we consider here only near-resonant radiating structures. Preliminary investigations have revealed that the use of the proposed method to design electrically small antennas [27] is still a challenge and would likely need further methods developments.

The rest of the article is organized into three major sections: problem formulation in the continuous case (Section II), specification of the numerical approach we use (Section II), and presentation of numerical results (Section IV).

\section{Problem Formulation}

A cross section of the domain of interest $\Omega_{\infty}$ is depicted in Fig. 1. Through a hole with radius $b$ in a ground plane located at $z=0$, a coaxial feed is entering the space $z>0$ vertically from below. The coaxial cable has a center core of radius $a$, a metallic shield of radius $b$, and is filled with a material with dielectric constant $\epsilon_{c}$ and permeability $\mu_{c}$. The domain $\Omega_{\infty}$ is defined as the union of the half space $z>0$ and the dielectric region of the coaxial cable for $-d<z \leq 0$, but the center core of the coaxial feed is excluded from $\Omega_{\infty}$. Included in the half space $z>0$ is an Ohmic region (conductivity $\sigma>0$ ) that constitutes the antenna. The annular surface, generated by a cross section at $z=-d$ through the dielectric part of the coaxial cable is denoted $\Gamma_{\text {coax }}$. The boundary $\partial \Omega_{\infty}$ consists of two parts: $\Gamma_{\text {coax }}$, where an incoming signal can be specified,

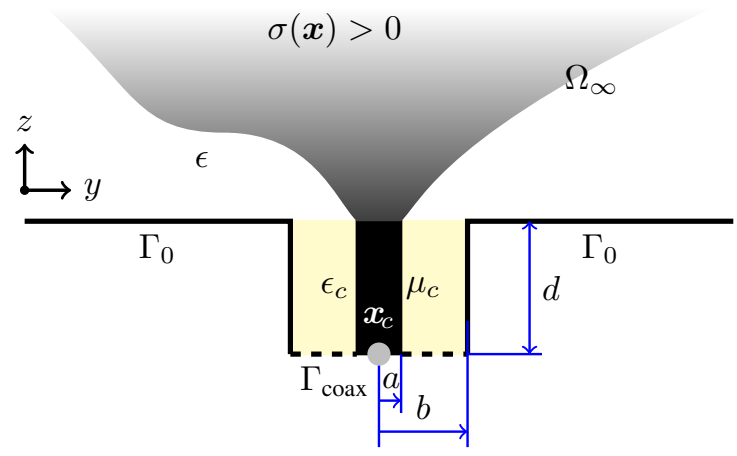

Fig. 1. Cross section in $y-z$ plane around the coaxial feed region. The domain $\Omega_{\infty}$ is the gray and white regions at $z>0$ (including the Ohmic region with $\sigma>0$ ) and the dielectric region of the coaxial feed. An input signal can be set at the annular region $\Gamma_{\text {coax }}$, located a distance $d$ below the ground plane.

and the perfect conductor $\Gamma_{0}$, which comprises the ground plane, the metallic shield, and the surface of the center core of the coaxial cable for $-d<z \leq 0$.

\section{A. Governing equations}

We consider the exterior initial-boundary-value problem

$$
\begin{array}{lr}
\frac{\partial}{\partial t} \mu \boldsymbol{H}+\nabla \times \boldsymbol{E}=\mathbf{0} & \text { in } \Omega_{\infty}, \text { for } t>0, \\
\frac{\partial}{\partial t} \epsilon \boldsymbol{E}+\sigma \boldsymbol{E}-\nabla \times \boldsymbol{H}=\mathbf{0} & \text { in } \Omega_{\infty}, \text { for } t>0, \\
\boldsymbol{n} \times \boldsymbol{E}=\mathbf{0} & \text { on } \Gamma_{0}, \text { for } t>0, \\
\boldsymbol{E}_{t}=\frac{V}{\log b / a} \frac{\boldsymbol{x}-\boldsymbol{x}_{\mathrm{c}}}{\left|\boldsymbol{x}-\boldsymbol{x}_{\mathrm{c}}\right|^{2}} & \boldsymbol{x} \in \Gamma_{\text {coax }}, t>0, \\
I=\int_{C_{r}} \boldsymbol{H} \cdot d \boldsymbol{l} & C_{r} \subset \Gamma_{\text {coax }}, \text { for } t>0, \\
V+Z_{\mathrm{c}} I=g(t) & \text { on } \Gamma_{\text {coax }}, \text { for } t>0, \\
\left.\boldsymbol{E}\right|_{t=0}=\mathbf{0}, & \\
\left.\boldsymbol{H}\right|_{t=0}=\mathbf{0}, & \\
\end{array}
$$

where $\mu, \epsilon$, and $\sigma$ are the permeability, the permittivity, and the conductivity of the medium, respectively; $Z_{\mathrm{c}}$ (defined in the Appendix) is the characteristic impedance of the coaxial cable; and $\boldsymbol{E}_{t}=\boldsymbol{E}-\boldsymbol{n}(\boldsymbol{E} \cdot \boldsymbol{n})$, where $\boldsymbol{n}$ is the outward unit normal vector. The system of equations (1) is solved for the electric field $\boldsymbol{E}$, the magnetic field $\boldsymbol{H}$, the potential difference $V$, and the core current $I$. The right-hand side of expression (1d) is the formula for the electric field in any transversal cross section in an infinite coaxial cable and will provide an approximation of the conditions at $\Gamma_{\text {coax }}$. Expression (1f) specifies a signal propagating in the positive $z$ direction. Equations (1) need to be complemented with far field boundary conditions to absorb the outgoing waves and possibly to impose incoming waves.

\section{B. Energy balance and the optimization problem}

We define a bounded sub-domain $\Omega=\Omega_{\infty} \cap B\left(R_{\mathrm{c}}, \mathbf{0}\right)$, where $B\left(R_{\mathrm{c}}, \mathbf{0}\right)$ is a ball centered at the origin and with a radius $R_{\mathrm{c}}$ large enough to encompass all parts of the antenna. We denote by $\Gamma_{\text {out }}=\Omega_{\infty} \cap \partial B\left(R_{\mathrm{c}}, \mathbf{0}\right)$ the boundary inside $\Omega_{\infty}$ of the encompassing sphere $\partial B\left(R_{\mathrm{c}}, \mathbf{0}\right)$. For signals with finite 


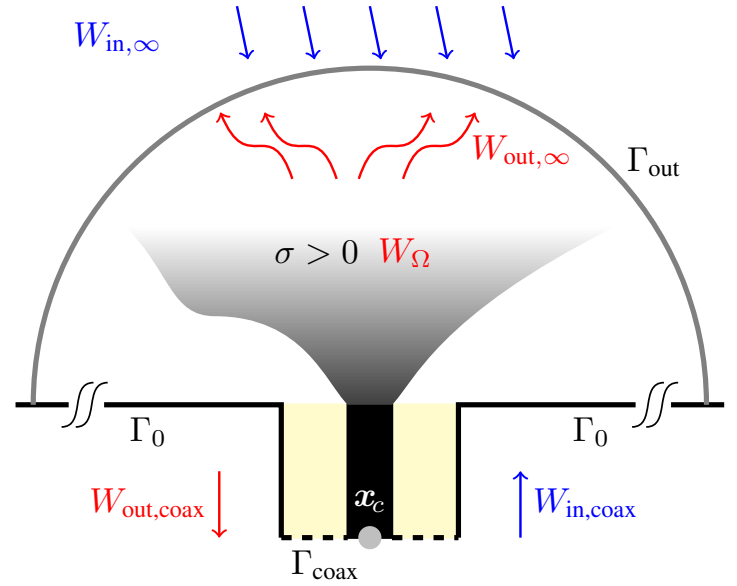

Fig. 2. An illustration of the system energy balance, where the sum of the incoming energies $W_{\mathrm{in}, \infty}+W_{\mathrm{in}, \mathrm{coax}}$ equal the sum of the outgoing energies $W_{\text {out }, \infty}+W_{\text {out }, \text { coax }}$ plus the energy loss $W_{\Omega}$.

extent in time, we derive from the system (1) the following energy balance

$$
W_{\text {in,coax }}+W_{\text {in, } \infty}=W_{\Omega}+W_{\text {out,coax }}+W_{\text {out }, \infty},
$$

in which

$$
\begin{aligned}
& W_{\text {in }, \text { coax }}=\frac{1}{4 Z_{\mathrm{c}}} \int_{0}^{T} g^{2} d t, \\
& W_{\text {out,coax }}=\frac{1}{4 Z_{\mathrm{c}}} \int_{0}^{T}\left(V-Z_{\mathrm{c}} I\right)^{2} d t, \\
& W_{\text {in, } \infty}=\frac{1}{4 Z_{0}} \int_{0}^{T} \int_{\Gamma_{\text {out }}}\left|\boldsymbol{E}_{t}+Z_{0} \boldsymbol{n} \times \boldsymbol{H}\right|^{2} d S d t, \\
& W_{\text {out }, \infty}=\frac{1}{4 Z_{0}} \int_{0}^{T} \int_{\Gamma_{\text {out }}}\left|\boldsymbol{E}_{t}-Z_{0} \boldsymbol{n} \times \boldsymbol{H}\right|^{2} d S d t, \\
& W_{\Omega}=\int_{0}^{T} \int_{\Omega} \sigma|\boldsymbol{E}|^{2} d V d t,
\end{aligned}
$$

where $Z_{0}=\sqrt{\mu_{0} / \epsilon_{0}}$ is the free space intrinsic impedance; $\mu_{0}$ and $\epsilon_{0}$ are the free space permeability and the permittivity; and $T$ is large enough to allow all fields to vanish. Fig. 2 illustrates the system energy balance; the incoming energy $W_{\text {in,coax }}+$ $W_{\text {in, } \infty}$ from the coaxial cable and exterior waves equals the ohmic losses in the antenna $W_{\Omega}$ plus the outgoing energy $W_{\text {out,coax }}$ through the coaxial cable and the wave energy $W_{\text {out, } \infty}$ exiting the domain.

Inspecting the energy balance (2), we see that there are two alternatives to set the energy sources for the current problem. The first is to use the coaxial cable to introduce the energy $W_{\text {in,coax }}$, and in this case an optimization problem can be formulated aiming at either maximizing the radiated energy $W_{\text {out }, \infty}$, as done by Erentok and Sigmund [24], or minimizing the reflected energy to the coaxial cable $W_{\text {out,coax }}$, as done by Nomura [18], where the complete optimization problem was lossless. However for the current work, we assume a lossy design medium, and a minimization of the reflected energy $W_{\text {out,coax }}$ can unfortunately be obtained through a maximization of the energy loss $W_{\Omega}$ inside the design domain.
The second alternative, and the one we choose, is to introduce the energy to the domain by using a set of external sources, which supply the incoming energy $W_{\text {in, } \infty}$. For this case, the antenna can be designed based on its receiving mode. Another motivation for this choice is that the full time history of the observed signal is needed to calculate the gradient of the objective function, as will be shown below. Observation of the received signal in the coaxial cable will require less memory than observation of the radiated waves $W_{\text {out }, \infty}$.

The optimization problem can conceptually be formulated as

$$
\underset{\sigma(\boldsymbol{x}) \in\left[\sigma_{\min }, \sigma_{\max }\right]}{\operatorname{maximize}} W_{\text {out,coax }}(\sigma)
$$

subject to the system of equations (1) with $g=0$ and a set of prescribed external sources that support the incoming electromagnetic waves. Moreover, $\sigma(\boldsymbol{x})$ is the conductivity distribution over the design domain $\Omega ; \boldsymbol{x}$ denotes a position in $\Omega ; \sigma_{\min }$ and $\sigma_{\max }$ represent the conductivities of a good dielectric and a good conductor, respectively. For the numerical experiments in this paper, we observed a very low sensitivity in the objective function to variations in conductivities below $\sigma_{\min }=10^{-4} \mathrm{~S} / \mathrm{m}$ or above $\sigma_{\max }=10^{5} \mathrm{~S} / \mathrm{m}$.

\section{Sensitivity analysis}

To solve problem (4) using gradient-based optimization algorithms, we need an expression for the derivatives of $W_{\text {out,coax }}$ with respect to changes in the conductivity distribution inside the design domain. Assuming that the conductivity $\sigma$ is perturbed by $\delta \sigma$, the corresponding first-order variation in the objective function $W_{\text {out,coax }}$ is

$$
\delta W_{\text {out,coax }}=\frac{1}{2 Z_{\mathrm{c}}} \int_{0}^{T}\left(V-Z_{\mathrm{c}} I\right)\left(\delta V-Z_{\mathrm{c}} \delta I\right) d t,
$$

where $\delta I$ and $\delta V$ are the first-order variations of the current and the potential difference inside the coaxial cable, respectively.

To find an explicit relation between $\delta W_{\text {out,coax }}$ and $\delta \sigma$, we need to know how $\delta I$ and $\delta V$ depend on $\delta \sigma$. These dependencies are resolved using the adjoint field method [17][20]. We use the system of governing equations (1) and apply the adjoint field method, summarized as follows. First, we differentiate system (1) with respect to the design variation $\delta \sigma$. Second, the differentiated equations corresponding to equation (1a) and equation (1b) are multiplied by the adjoint field vectors $\boldsymbol{H}^{*}$ and $\boldsymbol{E}^{*}$, respectively. Third, we integrate over the whole analysis domain $\Omega_{\infty}$ and through the time interval $(0, T)$, apply integration by parts, and utilize the boundary conditions and the far-field condition. Finally, after manipulating and rearranging the equations, we obtain an explicit relation between the conductivity perturbation and the first-order variation of the objective function in the form

$$
\delta W_{\text {out }, \text { coax }}=-\int_{\Omega} \int_{0}^{T} \boldsymbol{E}(T-t) \cdot \boldsymbol{E}^{*}(t) \delta \sigma d t d V,
$$


where $\boldsymbol{E}^{*}$ together with $\boldsymbol{H}^{*}, V^{*}$, and $I^{*}$ satisfy the following adjoint field problem,

$$
\begin{array}{lr}
\frac{\partial}{\partial t} \mu \boldsymbol{H}^{*}+\nabla \times \boldsymbol{E}^{*}=\mathbf{0} & \text { in } \Omega_{\infty}, \text { for } t>0, \\
\frac{\partial}{\partial t} \epsilon \boldsymbol{E}^{*}+\sigma \boldsymbol{E}^{*}-\nabla \times \boldsymbol{H}^{*}=\mathbf{0} & \text { in } \Omega_{\infty}, \text { for } t>0, \\
\boldsymbol{n} \times \boldsymbol{E}^{*}=\mathbf{0} & \text { on } \Gamma_{0}, \text { for } t>0, \\
\boldsymbol{E}_{t}^{*}=\frac{V^{*}}{\log b / a} \frac{\boldsymbol{x}-\boldsymbol{x}_{c}}{\left|\boldsymbol{x}-\boldsymbol{x}_{c}\right|^{2}} & \boldsymbol{x} \in \Gamma_{\text {coax }}, t>0, \\
I^{*}=\int_{C r} \boldsymbol{H}^{*} \cdot d \boldsymbol{l} & C r \subset \Gamma_{\text {coax }}, \text { for } t>0, \\
V^{*}(t)+Z_{\mathrm{c}} I^{*}(t)= & \text { for } t>0, \\
\left.\boldsymbol{E}^{*}\right|_{t=0}=\mathbf{0}, & \\
\left.\boldsymbol{H}^{*}\right|_{t=0}=\mathbf{0} . &
\end{array}
$$

The adjoint field problem only differs from the forward field problem (1) in the excitation expression (7f). We note that the right side of expression (7f) represents the outgoing signal inside the coaxial cable for the forward problem but reversed in time, and the left side represents the incoming signal inside the coaxial cable for the adjoint problem. Moreover, the adjoint system is excited only at the coaxial feed boundary $\Gamma_{\text {coax }}$, instead of through a set of far field sources like the forward problem.

\section{Filtering}

To be able to use gradient-based optimization, we allow the design conductivity $\sigma(\boldsymbol{x})$ to attain any value between $\sigma_{\min }$ and $\sigma_{\max }$. However, the intermediate values of $\sigma(\boldsymbol{x})$, will introduce energy losses to the system. For a certain intermediate value $\sigma_{\mathrm{m}}$ of the conductivity, which typically depend on the operating frequency band, the energy loss attains a maximum. This means that in order to maximize the outgoing signal $W_{\text {out,coax }}$, any optimization algorithm will drive the conductivity values away from $\sigma_{\mathrm{m}}$ and toward either $\sigma_{\mathrm{min}}$ or $\sigma_{\max }$. Thus, this optimization problem has the property of being self-penalized towards lossless designs. This property is in a sense useful, since in the end we want a lossless antenna. However, since the optimization problem is not convex, the optimization algorithm might be easily trapped into a poor local optimum. Moreover, a change of the conductivity at one point from $\sigma_{\min }$ to $\sigma_{\max }$ or vice versa, which means passing through $\sigma_{\mathrm{m}}$, will be considered by the optimization algorithm as a difficult barrier to cross.

To handle the problem of lossy intermediate designs in the framework of gradient-based optimization, we rely on filtering of the design variable, an approach that is classic in topology optimization, but for other reasons $[8, \S 1.3]$. Here, the main purpose of filtering the design conductivity is to regularize the strong self-penalization of the optimization problem by enforcing a certain amount of losses during the optimization process. Let $p(\boldsymbol{x}) \in[0,1]$ be the design variable that is actually updated by the optimization algorithm. This design variable is filtered to yield $\tilde{p}(\boldsymbol{x})=K_{R} * p(\boldsymbol{x})$, where the filtering is achieved through an integral operator $K_{R}$ with support in a disk of radius $R$. The filtered design variable is then mapped to the physical conductivity that is actually used in Maxwell's equations by

$$
\sigma(x)=10^{(9 \tilde{p}(x)-4)} .
$$

As long as the filter has nonzero radius, filtering the design variable $p(\boldsymbol{x})$ results in a "blurring" of the design conductivity $\sigma(\boldsymbol{x})$, which implies that the filter imposes a certain amount of energy losses inside the design domain. We start with an initial filter radius $R_{0}$ and successively reduce the radius by setting $R_{n+1}=\gamma R_{n}$, where $\gamma<1$, while performing a number of iterations of the optimization algorithm for each radius. We iterate until a selected convergence criterion based on the first-order necessary conditions is met; this typically takes about $10-20$ iterations. Thus, the algorithm progresses through a succession of less and less lossy designs until, for small values of the filter radius, the radiating element will almost entirely consist of elements with $\sigma$ being either $\sigma_{\text {min }}$ or $\sigma_{\max }$.

\section{NUMERICAL APPROACH}

As described in Section (II-A), problem (1) needs to be complemented with far field radiation conditions. For the numerical simulations, we use the uniaxial perfectly matched layer (UPML) [28] to simulate the open space radiation condition. Maxwell's equations can be written with the UPML parameters included as

$$
\begin{aligned}
\left(\frac{\partial}{\partial t}+\sigma^{p_{2}}\right) \boldsymbol{B}+\frac{1}{\mu} \nabla \times \boldsymbol{E} & =0, \\
\left(\frac{\partial}{\partial t}+\sigma^{p_{3}}\right) \boldsymbol{H}-\left(\frac{\partial}{\partial t}+\sigma^{p_{1}}\right) \boldsymbol{B} & =0, \\
\left(\frac{\partial}{\partial t}+\frac{\sigma}{\epsilon}\right) \boldsymbol{P}-\frac{1}{\epsilon} \times \boldsymbol{H} & =0, \\
\left(\frac{\partial}{\partial t}+\sigma^{p_{3}}\right) \boldsymbol{Q}-\frac{\partial}{\partial t} \boldsymbol{P} & =0, \\
\left(\frac{\partial}{\partial t}+\sigma^{p_{2}}\right) \boldsymbol{E}-\left(\frac{\partial}{\partial t}+\sigma^{p_{1}}\right) \boldsymbol{Q} & =0,
\end{aligned}
$$

with

$$
\begin{aligned}
& \sigma^{p_{1}}=\operatorname{diag}\left(\sigma_{x}^{p} / \epsilon_{0}, \sigma_{y}^{p} / \epsilon_{0}, \sigma_{z}^{p} / \epsilon_{0}\right), \\
& \sigma^{p_{2}}=\operatorname{diag}\left(\sigma_{y}^{p} / \epsilon_{0}, \sigma_{z}^{p} / \epsilon_{0}, \sigma_{x}^{p} / \epsilon_{0}\right), \\
& \sigma^{p_{3}}=\operatorname{diag}\left(\sigma_{z}^{p} / \epsilon_{0}, \sigma_{x}^{p} / \epsilon_{0}, \sigma_{y}^{p} / \epsilon_{0}\right),
\end{aligned}
$$

where $\sigma_{x}^{p}, \sigma_{y}^{p}$, and $\sigma_{z}^{p}$ represent fictitious conductivities that have nonzero values only inside the UPML layer, $\boldsymbol{E}$ and $\boldsymbol{H}$ are the primal electric and magnetic field vectors, and $\boldsymbol{B}$, $\boldsymbol{P}$, and $\boldsymbol{Q}$ are auxiliary field vectors used for the numerical implementation of the UPML. In the PML-free region all $\sigma^{p_{i}}$ are zeros, and equations (9) reduce to Maxwell's equations in free space $(1 \mathrm{a}-1 \mathrm{~b})$.

We use the FDTD method [26], [29] to solve numerically the time-domain Maxwell's equations. Based on the basic Yee cell, equations (9) are discretized to yield the standard FDTD update equations. We assume that the electric and magnetic fields are discretized at full and half time indices (leapfrog scheme), respectively. 


\section{A. Discrete objective function and Sensitivity analysis}

We formulate a discrete version of the objective function in problem (4) as

$$
W_{\text {out,coax }}^{\Delta}(\boldsymbol{\sigma})=\frac{1}{Z_{m}} \sum_{n=0}^{N}\left(V^{n+1}-\hat{Z}_{\mathrm{c}} I_{z}^{n+\frac{1}{2}}\right)^{2} \Delta t,
$$

where $V^{n+1}, I_{z}^{n+\frac{1}{2}}$, and $\hat{Z}_{\mathrm{c}}$ are the potential difference, the current, and the characteristic impedance of the discrete coaxial cable model (details are given in the Appendix); $Z_{\mathrm{m}}=\sqrt{\mu_{c} / \epsilon_{c}} ; N$ is the total number of time steps required for the simulation to reach the steady state condition, $\Delta t$ is the time step used in the FDTD method, and $\sigma$ is the conductivities located on each Yee edge inside the design domain. We derive the derivatives of the discrete objective function (11) with respect to the design conductivities $\sigma$. The whole derivation is done in the fully discrete case by using the standard FDTD update equations including the UPML parameters and the feeding model, described in the Appendix. Since the complete derivation is a lengthy process, we shortly point out the main steps and give the final expression that is used in the numerical calculations.

We start by perturbing the design conductivities by $\delta \boldsymbol{\sigma}$. The corresponding first-order variation of the discrete objective function is

$$
\begin{aligned}
& \delta W_{\text {out,coax }}^{\Delta}(\boldsymbol{\sigma})= \\
& \quad \frac{2}{Z_{m}} \sum_{n=0}^{N}\left(\delta V^{n+1}-\hat{Z}_{\mathrm{c}} \delta I_{z}^{n+\frac{1}{2}}\right)\left(V^{n+1}-\hat{Z}_{\mathrm{c}} I_{z}^{n+\frac{1}{2}}\right) \Delta t,
\end{aligned}
$$

where $\delta V^{n+1}$ and $\delta I_{z}^{n+\frac{1}{2}}$ are the first-order variations of the potential difference and the current inside the discrete coaxial cable, respectively. We use the adjoint field method to find an explicit relation between $\delta W_{\text {out,coax }}^{\Delta}$ and $\delta \boldsymbol{\sigma}$. We differentiate the FDTD discretization of equations (9) with respect to the design variables $\sigma$. Then, we form the scalar product of the differentiated equations with adjoint field vectors $\boldsymbol{B}^{*}, \boldsymbol{H}^{*}$, $P^{*}, Q^{*}, E^{*}$, that correspond to the direct field vectors $\boldsymbol{B}, \boldsymbol{H}$, $\boldsymbol{P}, \boldsymbol{Q}$, and $\boldsymbol{E}$, respectively. Next, we sum all equations over the whole analysis domain and through all time steps. Finally, applying summation by parts and rearranging the equations yield the three sets of equations that are described below.

First, we obtain a system of equations that corresponds to Maxwell's equations (9) with UPML parameters included; however, the FDTD discretization of this system has the adjoint electric and magnetic fields discretized at half and full time indices, respectively. We call this system the adjoint Maxwell's equations, and they constitute a FDTD discretization of the equations

$$
\begin{aligned}
\left(\frac{\partial}{\partial t}+\sigma^{p_{3}}\right) \boldsymbol{H}^{*}+\frac{1}{\mu} \nabla \times \boldsymbol{P}^{*} & =0, \\
\left(\frac{\partial}{\partial t}+\sigma^{p_{2}}\right) \boldsymbol{B}^{*}-\left(\frac{\partial}{\partial t}+\sigma^{p_{1}}\right) \boldsymbol{H}^{*} & =0, \\
\left(\frac{\partial}{\partial t}+\sigma^{p_{2}}\right) \boldsymbol{E}^{*}-\frac{1}{\epsilon} \nabla \times \boldsymbol{B}^{*} & =0, \\
\left(\frac{\partial}{\partial t}+\sigma^{p_{3}}\right) \boldsymbol{Q}^{*}-\left(\frac{\partial}{\partial t}+\sigma^{p_{1}}\right) \boldsymbol{E}^{*} & =0,
\end{aligned}
$$

$$
\left(\frac{\partial}{\partial t}+\sigma\right) \boldsymbol{P}^{*}-\frac{\partial}{\partial t} \boldsymbol{Q}^{*}=0 .
$$

Second, we obtain an expression for the excitation source to the adjoint Maxwell's equations in the form of the following discrete version of expression (7f),

$$
\begin{aligned}
& \left(V^{* n-\frac{1}{2}}+\hat{Z}_{\mathrm{c}} I_{z}^{* n-1}\right)= \\
& \left(V^{N-n+1}-\hat{Z}_{\mathrm{c}} I_{z}^{N-n+\frac{1}{2}}\right) \quad n=1, \ldots, N,
\end{aligned}
$$

where $V^{* n-\frac{1}{2}}$ and $I_{z}^{* n-1}$ are the discrete potential difference and the current in the coaxial cable for the adjoint problem, respectively.

Third, we obtain an expression for the gradient of the objective function with respect to the conductivity distribution

$$
\frac{\partial W_{\text {out,coax }}^{\Delta}}{\partial \sigma_{i}}=-\Delta^{3} \sum_{n=1}^{N} E_{i}^{N-n} \frac{P_{i}^{* n-\frac{1}{2}}+P_{i}^{* n+\frac{1}{2}}}{2} \Delta t,
$$

where $i$ is the index for an arbitrary Yee edge inside the design domain and $\Delta$ is the spatial discretization step (assuming a uniform spatial discretization).

Equations (13) can be seen as a version of Maxwell's equations in the primal field vectors $\boldsymbol{P}^{*}$ and $\boldsymbol{B}^{*}$, where the auxiliary field vectors $H^{*}, E^{*}$, and $Q^{*}$ are used for the implementation of the UPML. The auxiliary equations are arranged in a different order compared to the auxiliary equations of the forward problem (9). Inside the PML-free region (i.e., when $\sigma^{p_{i}}=0$ ), the discretization of the adjoint and the forward equations are identical, and the same FDTD code can be used to update the primal fields.

Expression (15) yields exact derivatives of the objective function (11), up to roundoff, when solutions to the discrete form of equations (13) are used. However, a derivative computation based on these equations requires a separate FDTD code for the adjoint equations that will be different from the forward FDTD code only in the implementation of the UPML. To avoid this complication, we instead used the UPML implementation of the forward equations also for the adjoint equations. We verified the derivatives calculated by expression (15), with the modified treatment of the UPML, against derivatives approximated with finite differences, exploiting Richardson extrapolation [30] for improved accuracy. These experiments achieved 7 digits precision matching between the two methods. We regarded this accuracy as sufficient for the purpose of optimization.

\section{B. Discrete optimization problem}

Including the filtering and mapping of the design variables (Section II-D), the discrete version of the optimization problem (4) can be written as

$$
\underset{\boldsymbol{p} \in \mathcal{A}}{\operatorname{maximize}} W_{\mathrm{out}, \mathrm{coax}}^{\Delta}(\boldsymbol{\sigma}(\tilde{\boldsymbol{p}}(\boldsymbol{p}))),
$$

where $\mathcal{A}=\left\{\boldsymbol{p} \in \mathbb{R}^{M} \mid \quad p_{i} \in[0,1] \quad \forall i\right\}$ is the set of admissible design variables.

All design variables are subject to simultaneous updates by the optimization algorithm. These updates are based on the derivative expression (15). We use the globally convergent 
method of moving asymptotes (GCMMA) by Svanberg [31], to update the design variables. By construction, the GCMMA naturally imposes the box constraints in the definition of the approximating functions at each iteration. Moreover, the GCMMA is also in other respects well suited to the mathematical structure of topology optimization problems [8, §1.2].

The complete optimization process is illustrated by the flowchart in Fig. 3. The process starts with a uniform initial distribution of the design variables $\boldsymbol{p}$ over the design domain $\Omega$. We set an initial radius $R_{0}$, filter the design variables, and compute the physical conductivities $\sigma$ as described in Section II-D. Then, the FDTD method is used to solve the forward problem. For each time step, we store the forward electric field $E_{i}$ for each edge $i$ inside the design domain and the outgoing signal $h_{\mathrm{c}}^{n+1}$ from the coaxial cable. We also use the signal $h_{\mathrm{c}}^{n+1}$ to compute the objective function (11). For the forward problem, only far field sources are used as excitation sources. Next, the optimization process calls the FDTD method another time to calculate and store the adjoint field $P_{i}^{*}$ for each edge $i$ inside the design domain and for each time step; however, here the problem is excited only at the coaxial cable port by using expression (14). The stored electric field $E_{i}$ and the adjoint field $P_{i}^{*}$ are used to evaluate the gradient with respect to the conductivity $\sigma$ by expression (15). By the chain rule, the gradient with respect to the design variables $\boldsymbol{p}$ is

$$
\frac{\partial W_{\mathrm{out}, \mathrm{coax}}^{\Delta}(\boldsymbol{\sigma}(\tilde{\boldsymbol{p}}(\boldsymbol{p})))}{\partial \boldsymbol{p}}=\frac{\partial \tilde{\boldsymbol{p}}}{\partial \boldsymbol{p}} \frac{\partial \boldsymbol{\sigma}}{\partial \tilde{\boldsymbol{p}}} \frac{\partial W_{\mathrm{out}, \mathrm{coax}}^{\Delta}(\boldsymbol{\sigma})}{\partial \boldsymbol{\sigma}} .
$$

The first two factors on the right side of expression (17) are transposes of Jacobians associated with the filter and the conductivity mapping introduced in Section II-D. Then, a convergence criterion based on the first-order necessary condition is tested. The norm of the first-order necessary condition is compared with a reference value, which is recorded after a fixed number of iterations (in the numerical experiments, we use 6 iterations). The iterations are terminated when the norm of the first-order necessary condition is below $50 \%$ of the reference value. If the convergence criterion is not satisfied, the optimization process continues to a new cycle where the GCMMA algorithm use the gradient and the objective function values to update the design variables. At each cycle, the GCMMA might evaluate the objective function a few additional times to find a suitable updates that satisfy a sufficient improvement of the objective function. If the convergence criterion is reached but the filter radius is greater than $\tilde{\Delta}=\Delta / \sqrt{2}$, the minimal distance between two conductivity components in the Yee cell, the radius is reduced, $R_{n+1}=\gamma R_{n}$, and a new cycle starts. Finally, the optimization process terminates if the convergence criterion is satisfied and the filter radius is smaller than $\tilde{\Delta}$. Typically, the final design consists of conductivities that either have $\sigma_{\min }$ or $\sigma_{\max }$. In the numerical experiments, when we evaluate the performance of the obtained design, physical conductivities below $10^{-3} \mathrm{~S} / \mathrm{m}$ are mapped to $\sigma=0 \mathrm{~S} / \mathrm{m}$ while conductivities above that value are mapped to $\sigma=5.8 \times 10^{7} \mathrm{~S} / \mathrm{m}$.

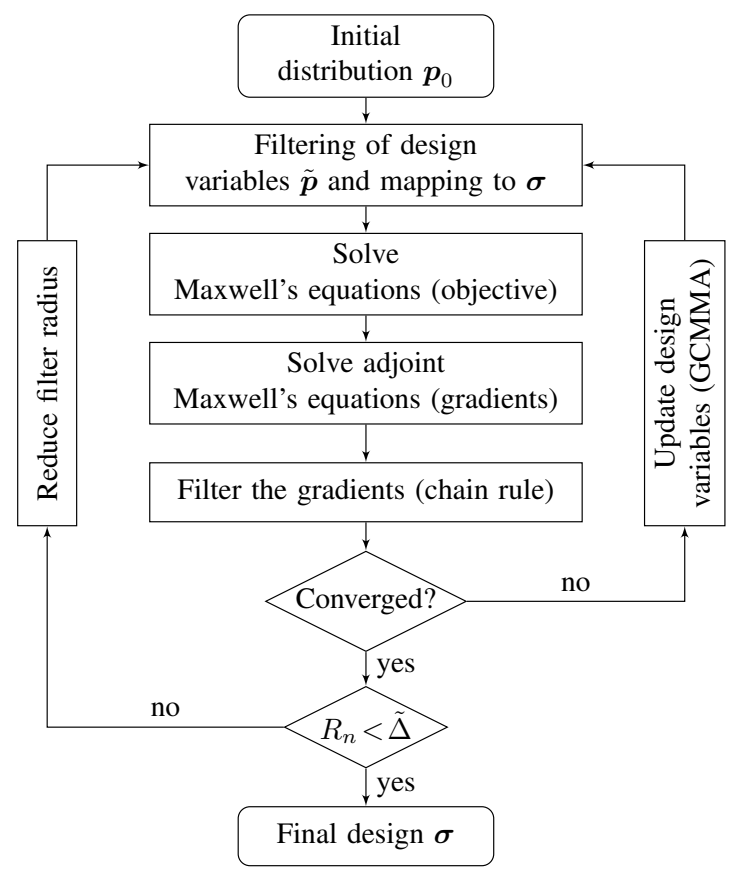

Fig. 3. A flowchart of the optimization process.

\section{NUMERICAL RESULTS AND DISCUSSION}

In transmission mode, antennas transmit the incoming energy from the feed lines to the surrounding space with a certain directivity. Since the current work is based on designing antennas based on their receiving mode, the distribution of the far field sources are expected to have a significant impact on the solution of problem (16). In the numerical experiments, we observe that an increase in the number of the far field sources around the design domain enables the optimization algorithm to find designs that occupy a smaller design space and have a better performance, in terms of the antenna reflection coefficient, over the frequency band of interest. For the monopole design, we use four plane waves that propagate parallel to the ground plane and normal to the four sides of the FDTD cubical grid. For the microstrip case, an additional plane wave propagates from the top side of the cubical grid towards the patch area. In both cases, the plane waves are synchronized to arrive to the feeding point at the same time. To cover a certain frequency band, we use a truncated sinc pulse modulated to the center frequency of that band.

In the first announcement of this work [32], we presented a design of a UWB monopole antenna based on linearly polarized field excitation. In the current work, the designs are based on a set of far field sources that essentially radiate circularly polarized plane waves. We use the total-field scattered-field formulation to implement the wave excitation in the FDTD method [26], where we use two signals orthogonal in space and with 90 degree phase shift to modulate the sinc pulse mentioned above. At the ground plane surface, the boundary condition does not allow for an implementation of a circularly polarized plane wave that propagates parallel to the surface. However, away from the ground plane, this wave excitation allows both vertical and horizontal polarized 
waves in the design domain, which allows the optimization algorithm to maximize the received energy regardless of the signal's polarization. We emphasize that this objective can easily be changed to design for specific far field characteristics by modifying either the wave polarization or the incidence direction.

For all numerical experiments, we use 10 cells of the UPML, 10 cells free space separation between the UPML and the simulated designs, $\Delta=0.75 \mathrm{~mm}$, and $\Delta t$ set to 0.95 times the Courant limit. The FDTD code is implemented to run on Graphics Processing Units (GPUs), using the parallel computing platform CUDA (https://developer.nvidia.com/what-cuda). All numerical experiments are performed on an nVidia GeForce GTX 285 installed on a node with two AMD Opteron 8431 running at $2.4 \mathrm{GHz}$ with $32 \mathrm{~GB}$ shared memory. We use double precision floating point arithmetic for all calculations. The FDTD simulation time, for the forward or the adjoint field solution, varies between 1-3 minutes depending on the excitation signal bandwidth and the dimensions of the computational domain.

To compute the gradient given in expression (15), the proposed algorithm requires memory that is proportional both to the number of the Yee edges in the design domain and the number of time steps used in the simulation. In this work, the gradient calculation requires $1.5 \mathrm{~GB}$ and $3.3 \mathrm{~GB}$ of memory for the UWB monopole and the microstrip patch, respectively. For design problems that would require even more storage, the memory requirements can be significantly reduced by using checkpointing techniques [33].

\section{A. UWB monopole design}

In this section, we design the radiating patch of a thin planar monopole. We consider a design domain with area $75 \times 75 \mathrm{~mm}^{2}$, located $0.75 \mathrm{~mm}$ above an infinite simulated ground plane, which is fed by a $50 \mathrm{Ohm}$ coaxial cable at the center of its bottom side. In the FDTD grid, we model the design domain as a single layer discretized into $100 \times 100$ Yee cell faces, which gives a design space consisting of 20, 200 design variables (one conductivity component for each Yee edge). The design domain is expected to have an effective mesh-dependent finite thickness $\tau_{\text {eff }} \approx 0.2 \Delta$ [34], which in our case is approximately $0.15 \mathrm{~mm}$. We solve problem (16) to maximize the received signal from the far field sources, mentioned above, over the frequency band 1-10 GHz. The design starts from a uniform distribution of conductivities that corresponds to a value 0.7 for each element in $p$. The initial filter radius $R_{0}$ is $15 \mathrm{~mm}$, and a filter decrease coefficient $\gamma=0.75$ is used. To avoid disconnection between the inner probe of the coaxial cable and the design domain, especially when the radius of the filter changes, the probe is extended into the design domain by a length equal to the filter radius.

The progress of the filtered design variables $\tilde{p}$ over a number of optimization cycles is shown in Fig. 4. The optimization process starts with a large filter radius that imposes a thick layer of intermediate values of the design variable. As the optimization process proceeds, the filter radius decreases and the thickness of this layer diminishes. By the end of the

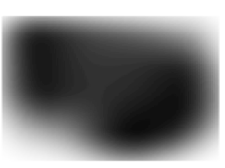

(a) cycle 1

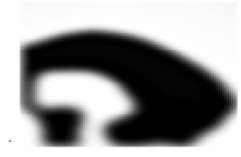

(d) cycle 46

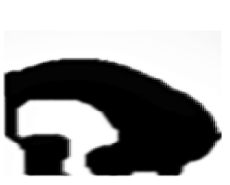

(g) cycle 91

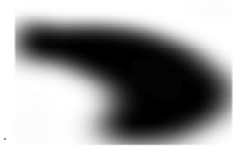

(b) cycle 16

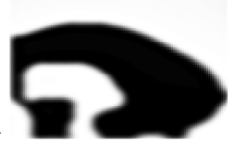

(e) cycle 61

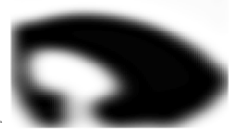

(c) cycle 31

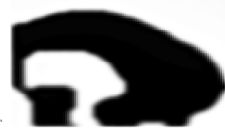

(f) cycle 76

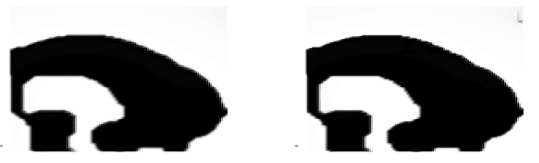

(h) cycle 106

$0.4 \quad 0.6$

0.8

Fig. 4. The filtered design variables at a number of optimization cycles for the design of the UWB monopole based on a circularly polarized plane wave excitation. Black color means $\sigma_{\max }$ and white color means $\sigma_{\min }$.

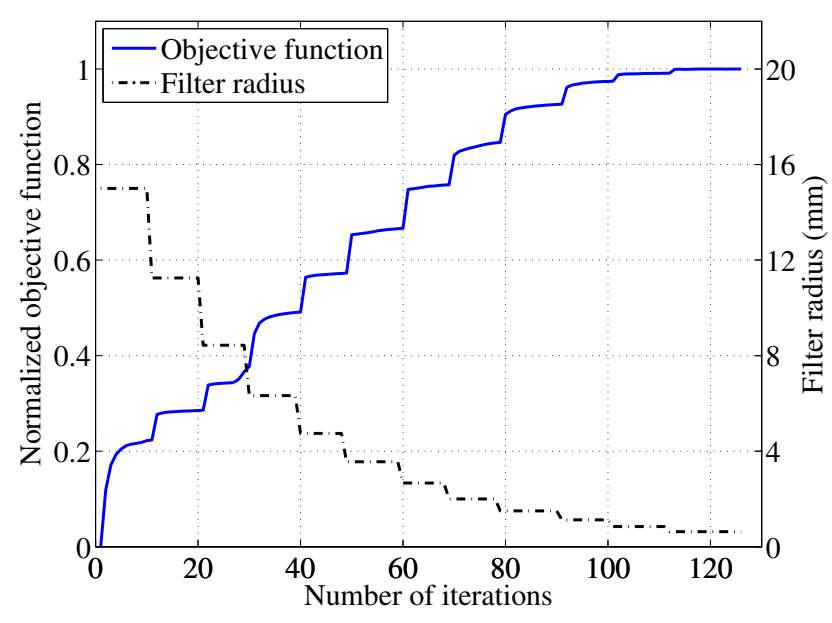

Fig. 5. Progress in the normalized objective function and the filter radius during the design of the planar monopole.

optimization process, the filtered design variables converge to values that correspond to $\sigma_{\max }$ (black color) or to $\sigma_{\min }$ (white color). Fig. 5 shows the progress of the normalized objective function during the optimization process. As can be noticed, for a fixed filter radius, there is only a small increase in the received energy since the filtering process forces the energy loss to dominate. When the filter radius is reduced, the optimization algorithm updates the conductivities away from the lossy intermediate values in order to maximize the received energy, which leads to a sudden increase in the received energy.

The optimization algorithm required 126 cycles to converge to the final optimized design shown in Fig. 6 . The final design uses around $75 \times 60 \mathrm{~mm}^{2}$ of the available design domain $75 \times 75 \mathrm{~mm}^{2}$. When the filter radius decreases to very small values, tiny groups of isolated pixels containing conductive 


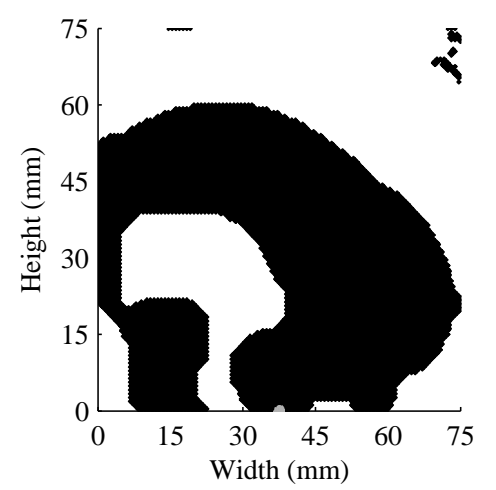

Fig. 6. The final design of the UWB planar monopole. Design domain size $75 \times 75 \mathrm{~mm}^{2}(20,200$ design variables). Coaxial probe connected at $(37.5$, 0) $\mathrm{mm}$.

material may appear, as can be seen in the upper right corner of the design domain in Fig. 6. Fortunately, these groups are typically small, appear far away from the feeding point, and have negligible effect on the final performance. A remedy to this issue in a general topology optimization problem is to keep the filter radius above a certain value [8], however as mentioned earlier, for topology optimization of lossy media the final filter radius should be zero to avoid losses.

The reflection coefficient of the final design is calculated by our FDTD code, and the result is shown in Fig. 7 (solid line). As a verification of our implementation, we plot in the same figure the result obtained with the CST Microwave Studio software (dashed line), employing adaptive mesh refinement and modelling the monopole as a conductive sheet that has a thickness $0.15 \mathrm{~mm}$ and a conductivity $5.8 \times 10^{7} \mathrm{~S} / \mathrm{m}$. We include also the radiation efficiency computed by the CST package in the same figure. A source for the slight difference between the two simulation results could be differences in the geometry description between the two methods. The reflection coefficient of the final design is below $-10 \mathrm{~dB}$ over the frequency band $1.23-9.75 \mathrm{GHz}$, and the computed radiation efficiency is on average above $99.6 \%$ over the target frequency band.

Inspecting Fig. 6, we can see that the final design hits the left boundary of the design domain. This implies that the design wants to grow to the left but it is bounded by the physical dimensions of the design domain. We decide to test a larger design domain with size $135 \times 75 \mathrm{~mm}^{2}$, which is discretized into $180 \times 100$ Yee cell faces. The optimization problem has 36, 280 unknowns and is solved by the optimization algorithm in 130 iterations. The new design, shown in Fig. 8, consists of two disconnected parts: an outer loop and a conductive island to the left of the feed point. The new design grows to the left, utilizes more space, but still hits the left boundary; however it does not grow at all to the right side of the design domain. The growth of the outer loop improves the reflection coefficient at the lower side of the frequency band, as can be seen in Fig. 9, where the lower limit of the reflection coefficient has moved to $1.0 \mathrm{GHz}$, whereas the upper limit is still at $9.7 \mathrm{GHz}$. When removing the

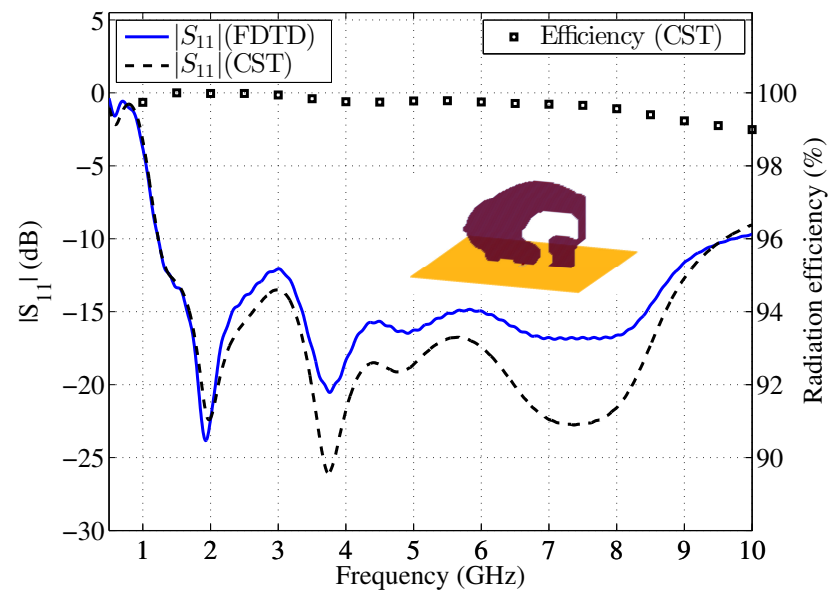

Fig. 7. The reflection coefficient, $\left|S_{11}\right|$, and the radiation efficiency of the designed UWB planar monopole.

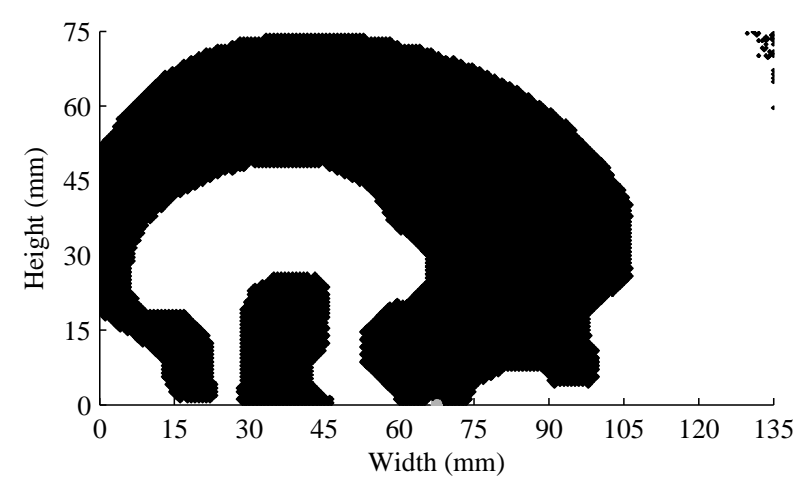

Fig. 8. The final design of the UWB planar monopole. Design domain size $135 \times 75 \mathrm{~mm}^{2}(36,280$ design variables $)$. Coaxial probe connected at $(67.5$, 0) $\mathrm{mm}$.

disconnected conductive island near the feed, the performance of the optimized monopole deteriorates somewhat, as can be seen in Fig. 9. This observation emphasizes the usefulness of the disconnected conductive island, build by the optimization algorithm, to improve the overall performance of the monopole inside the objective frequency band.

\section{B. Microstrip antenna design}

To demonstrate the general applicability of the proposed design approach, we also consider the design of the metallic radiating patch of a microstrip antenna. First, the patch area is designed to radiate in a single frequency band centered around $1.5 \mathrm{GHz}$ with $0.2 \mathrm{GHz}$ bandwidth. Second, the same design domain is used for achieving a dual-band operation with the frequency bands centered around 1.5 and $2.0 \mathrm{GHz}$, which cover the GPS L1 band $(1.575 \mathrm{GHz})$ and the UMTS band $(1.92-2.17 \mathrm{GHz})$, respectively. As in the monopole case, we use a design domain of $75 \times 75 \mathrm{~mm}^{2}$, and discretize it into $100 \times 100$ Yee cell faces (i.e., 20,200 design variables). We consider a substrate with $6 \mathrm{~mm}$ height, 2.62 dielectric constant and 0.001 loss tangent at $2 \mathrm{GHz}$. The design space (radiating 


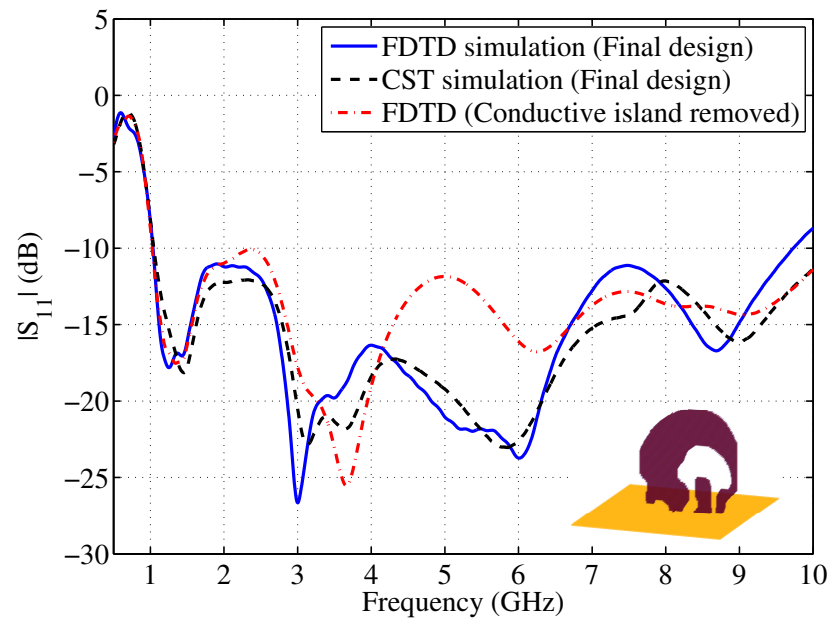

Fig. 9. The reflection coefficient, $\left|S_{11}\right|$, of the designed UWB planar monopole with and without the conductive island near the feed point.

patch) is positioned on an infinite simulated ground plane and fed by $50 \Omega$ coaxial cable. The design process uses an initial conductivity distribution that corresponds to a value 0.5 for each element in $\boldsymbol{p}$, an initial filter radius $R_{0}=15 \mathrm{~mm}$, and a filter decrease coefficient $\gamma=0.75$.

Fig. 10 shows the final conductivity distribution of two designs that are designed to maximize the energy received by the microstrip antenna at $1.5 \mathrm{GHz}$. In the design to the left, the coaxial cable is connected at $(37.5,37.5) \mathrm{mm}$, while in the design to the right, the coaxial cable is connected at $(18.75,37.5) \mathrm{mm}$. The feed position is marked by gray circle in the figure. The optimization algorithm converged to the final designs after 155 and 118 iterations, respectively. The reflection coefficient of the final antennas, calculated by the FDTD method and verified with the CST package, and the radiation efficiency (computed with CST package) are shown in Fig. 11 and Fig. 12, respectively. In the CST computations, the antennas are assigned a thickness of $0.15 \mathrm{~mm}$ and a conductivity of $5.8 \times 10^{7} \mathrm{~S} / \mathrm{m}$. As can be noticed, we chose the feed positions close to the center of the design domain, which gives the algorithm more freedom to choose which direction to distribute the conductivity. In both cases, the optimization algorithm is able to find designs that maximize the received energy over the frequency band of interest, $1.4-1.6 \mathrm{GHz}$; however the reflection coefficient of the optimized designs are only below $-10 \mathrm{~dB}$ in the frequency band $1.46-1.59 \mathrm{GHz}$ for the first design and 1.49-1.64 GHz for the second design.

We notice that for narrow band designs, like the current case, the final optimized designs generally tend to have a better performance (i.e., $\left|S_{11}\right|<-10 \mathrm{~dB}$ ) at the higher side of the frequency band of interest. This observation can be explained as follows. The optimization algorithm is based on maximizing the received energy $W_{\text {out,coax }}$, which is equivalent to minimizing the energy losses $W_{\Omega}$ and the reflected signal $W_{\text {out }, \infty}$ (energy balance (2)). The presence of intermediate conductivities makes the design domain dissipative. The energy losses (the wave attenuation constant) will be

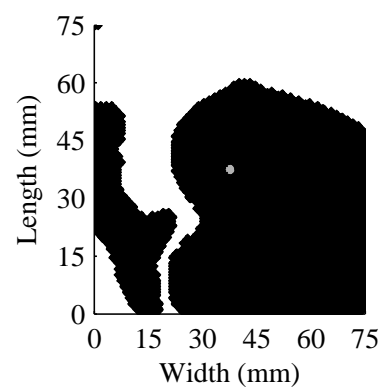

(a)

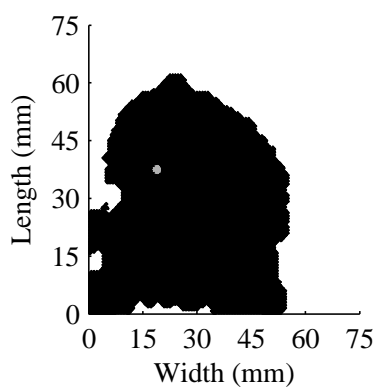

(b)
Fig. 10. The final conductivity distributions over the patch area that maximize the energy received over a frequency band $0.2 \mathrm{GHz}$ centered at $1.5 \mathrm{GHz}$. The coaxial feed located at (a) $(37.5,37.5) \mathrm{mm}$ and (b) $(18.75,37.5) \mathrm{mm}$.

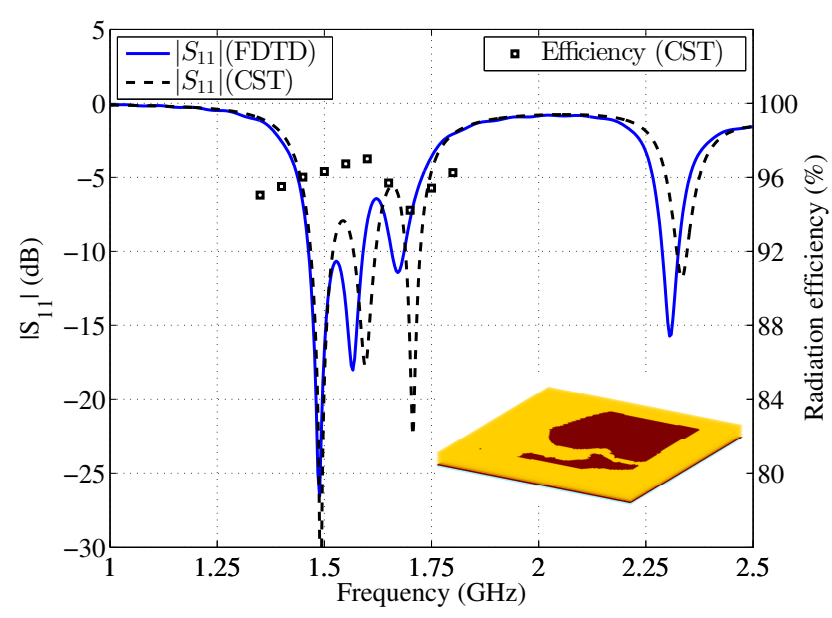

Fig. 11. The reflection coefficient, $\left|S_{11}\right|$, and the radiation efficiency of the optimized patch area that is connected to the coaxial cable at $(37.5,37.5) \mathrm{mm}$ inside the design domain.

proportional to the frequency of the incident waves [35]. Thus, the optimization process will implicitly focus on the higher frequencies in the incident waves to reduce the energy losses. This issue becomes more severe later when we optimize for dual band operation. One way to circumvent this phenomenon for the single narrow band case, and to obtain a frequency response closer to the target frequency band, is simply to lower the center frequency of the incident waves. Fig. 13 shows a different design obtained after 113 iterations by the optimization algorithm, when the center frequency of the incident waves is lowered to $1.4 \mathrm{GHz}$. As can be noted, the operational frequency band where the reflection coefficient is below $-10 \mathrm{~dB}$ (i.e., $1.38-1.51 \mathrm{GHz}$ ), is lowered almost with the same amount as the change in the center frequency of the excitation signals, that is, $0.1 \mathrm{GHz}$.

As a final case study, we design the patch area to radiate in two frequency bands centered around $1.5 \mathrm{GHz}$ and $2 \mathrm{GHz}$, respectively, with $0.2 \mathrm{GHz}$ bandwidth for each band. We use the same settings as for the single frequency experiment with the coaxial cable connected to the design domain at (18.75, 37.5) $\mathrm{mm}$. To handle the two band operation, a new objective 


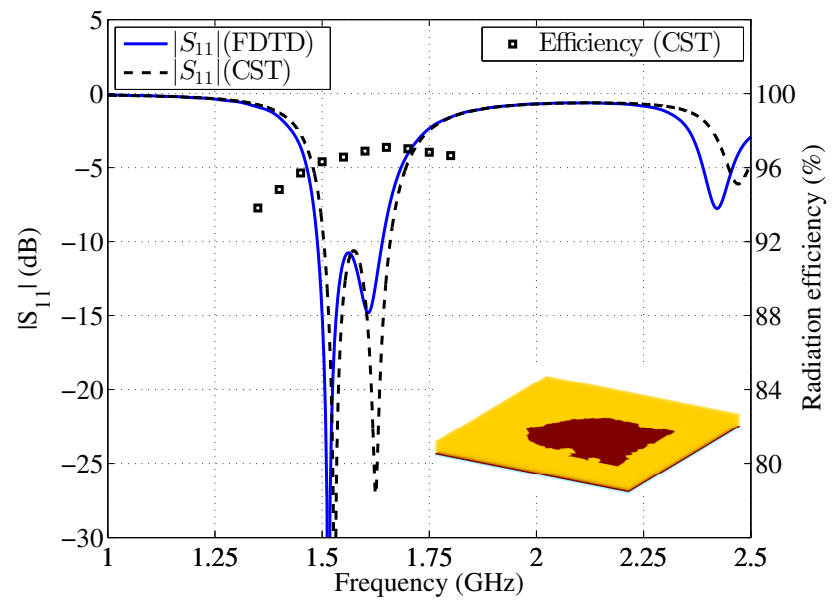

Fig. 12. The reflection coefficient, $\left|S_{11}\right|$, and the radiation efficiency of the optimized patch area that is connected to the coaxial cable at $(18.75$, 37.5) $\mathrm{mm}$ inside the design domain.

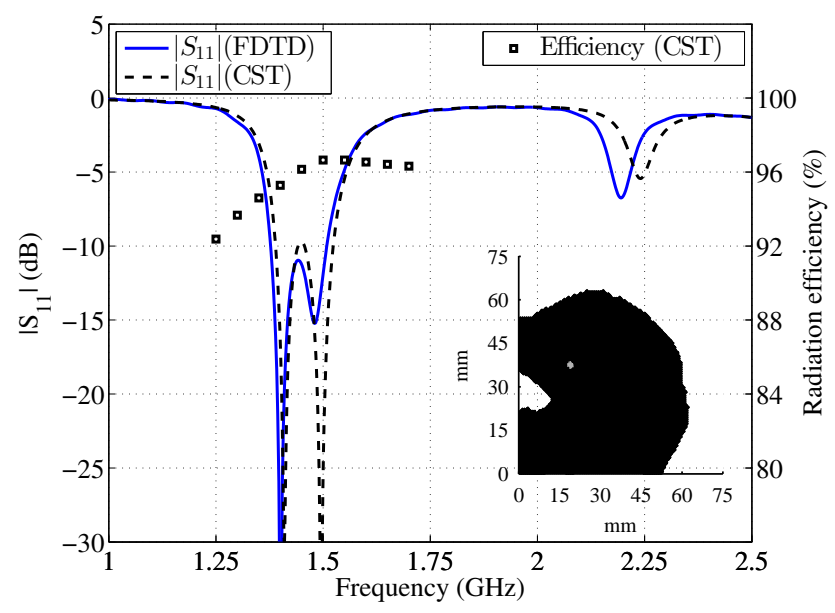

Fig. 13. The conductivity distribution, the reflection coefficient, and the radiation efficiency of the optimized patch area when the center frequency of the incident waves is lowered to $1.4 \mathrm{GHz}$. The coaxial cable is connected at $(18.75,37.5) \mathrm{mm}$.

function is formulated based on a combination of the energies $W_{\text {out,coax }}^{\Delta 1}$ and $W_{\text {out,coax }}^{\Delta 2}$ that are received from the first and the second frequency band, respectively. We formulate the objective function in terms of a positive exponent $\rho$, as follows,

$$
\underset{\boldsymbol{p} \in \mathcal{A}}{\operatorname{maximize}} \sum_{i=1}^{2}\left|W_{\text {out,coax }}^{\Delta i}(\boldsymbol{\sigma}(\tilde{\boldsymbol{p}}(\boldsymbol{p})))\right|^{\rho} .
$$

First, we solve the optimization problem (18) with $\rho=1$, which means that the objective function is simply the linear combination of $W_{\text {out,coax }}^{\Delta 1}$ and $W_{\text {out,coax }}^{\Delta 2}$. Fig. 14 shows the final design (obtained after 115 iterations) together with the corresponding reflection coefficient and radiation efficiency. The obtained design has a reflection coefficient below $-10 \mathrm{~dB}$ over the frequency band $1.88-2.13 \mathrm{GHz}$. However, the reflection coefficient is almost $0 \mathrm{~dB}$ around $1.5 \mathrm{GHz}$. As mentioned earlier, the dissipative characteristics associated with the in-

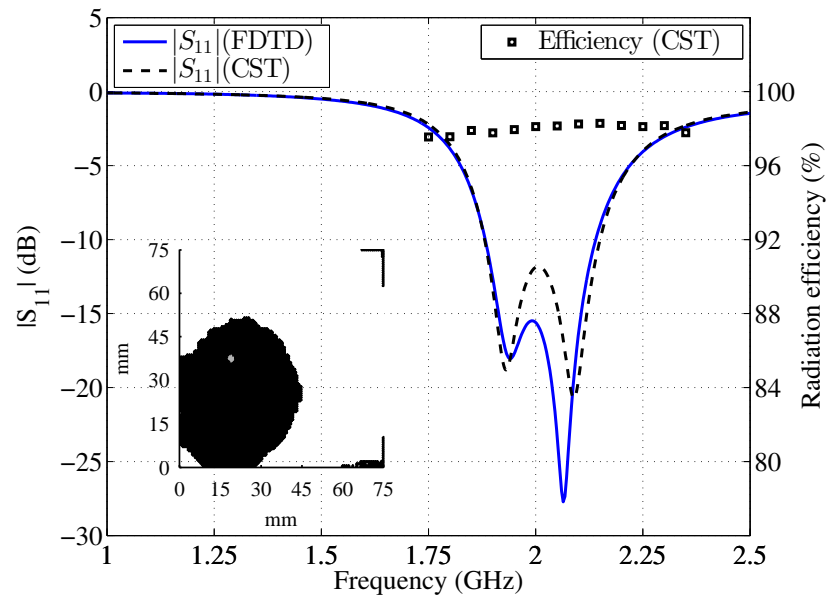

Fig. 14. The conductivity distribution, the reflection coefficient, and the radiation efficiency of the optimized patch area, when optimizing for two frequency bands centered around $1.5 \mathrm{GHz}$ and $2 \mathrm{GHz}$ with $0.2 \mathrm{GHz}$ bandwidth for each band, $\rho=1$, and the coaxial feed is located at $(18.75,37.5) \mathrm{mm}$.

termediate values of the design variables implicitly drive the optimization algorithm to focus on the higher frequency band, and the lower band is almost neglected.

The optimization problem (18) can be modified to obtain a balance between the two frequency bands by using $\rho<1$; that is, by using nonlinear combinations of $W_{\text {out,coax }}^{\Delta 1}$ and $W_{\text {out,coax }}^{\Delta 2}$. In this case, term $i$ in the gradient of the objective function in (18) will be scaled by $\left(W_{\text {out,coax }}^{\Delta i}\right)^{\rho-1}$, which means that the lower received energy will have more contribution to the total gradient, and as the value of $\rho$ decreases, the bias towards the lower received energy increases. Fig. 15 shows the conductivity distribution, the reflection coefficient, and the radiation efficiency of the final design obtained by the optimization algorithm (after 117 iterations) when we set $\rho=1 / 2$. The reflection coefficient of the obtained design satisfies the dual band operation, where the reflection coefficient is below $-10 \mathrm{~dB}$ in the frequency bands $1.51-1.55 \mathrm{GHz}$ and 1.98 $2.10 \mathrm{GHz}$. As can be noted, inside each frequency band, the performance is still biased toward the higher frequency edge, as in the single band design case.

\section{SUMMARY AND CONCLUSION}

We propose an approach to carry out gradient-based topology optimization for the design of metallic antennas based on the distribution of the material conductivity in a given domain. The antenna model is formulated using the 3D Maxwell's equations in time domain. In addition, we introduce a simple and efficient way to model a coaxial cable feed. The FDTD method is used to numerically solve the problem. The conductivities associated with each Yee edge in the design domain are considered as design variables, which implies a very detailed parameterization of the complete design domain. Solving Maxwell's equations in time domain enables a simultaneous design over frequency bands. The design conductivities are assumed to vary continuously between two extreme values. 


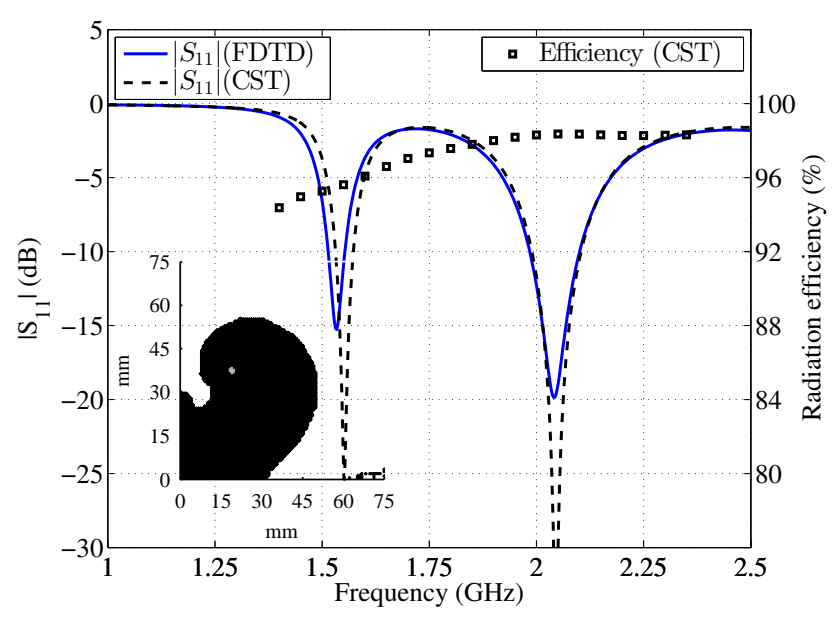

Fig. 15. The conductivity distribution, the reflection coefficient, and the radiation efficiency of the optimized patch area that radiates over two frequency band centered around $1.5 \mathrm{GHz}$ and $2 \mathrm{GHz}$ with $0.2 \mathrm{GHz}$ bandwidth for each band, $\rho=1 / 2$, and the coaxial feed is located at $(18.75,37.5) \mathrm{mm}$.

The intermediate values introduce energy losses to the design problem. To handle these losses, the design is based on the antenna receiving mode and a filtering approach is introduced. The technique is utilized to design the radiating patch of both planar monopole antennas and microstrip antennas. The technique gives fascinating shapes without any prior assumptions about details in the designs. We present results that focus on improving the antenna reflection coefficient. However, the approach can be modified for other objectives, such as the control of the directivity and the field polarizations.

\section{APPENDIX}

\section{DISCRETE COAXIAL CABLE MODEL}

A common approach to model a coaxial feed line with the FDTD method is either to fully model the coaxial cable, to use a separate 1D grid, or to use some quasi-static approximations [36]-[38]. Here, we propose a simple and efficient model of the coaxial cable feed that can be used with the FDTD method. This model has the advantage of satisfying a similar symmetry property as the coaxial feeds in equations (1) and (7): the discrete Maxwell's and the discretely-derived adjoint Maxwell's equations will have precisely the same feeding models.

To motivate our feeding model, recall that inside the coaxial cable, assuming that only TEM waves are supported, the transverse electric and magnetic fields $\tilde{\boldsymbol{E}}$ and $\tilde{\boldsymbol{H}}$ satisfy

$$
\frac{\partial}{\partial t}\left(\sqrt{\epsilon_{c}} \tilde{\boldsymbol{E}} \mp \sqrt{\mu_{c}} \boldsymbol{k} \times \tilde{\boldsymbol{H}}\right) \pm c \frac{\partial}{\partial z}\left(\sqrt{\epsilon_{c}} \tilde{\boldsymbol{E}} \mp \sqrt{\mu_{c}} \boldsymbol{k} \times \tilde{\boldsymbol{H}}\right)=0,
$$

where $\boldsymbol{k}$ is a unit vector in the positive $z$ direction; $\mu_{c}$ and $\epsilon_{c}$ are the permeability and the permittivity inside the coaxial cable; and $c=1 / \sqrt{\mu_{c} \epsilon_{c}}$. Expression (19) is a transport equation for

$$
\tilde{\boldsymbol{W}}_{\mathrm{c}}^{ \pm}=\sqrt{\epsilon_{c}} \tilde{\boldsymbol{E}} \mp \sqrt{\mu_{c}} \boldsymbol{k} \times \tilde{\boldsymbol{H}} .
$$

The characteristic variables $\tilde{\boldsymbol{W}}_{\mathrm{c}}^{+}$and $\tilde{\boldsymbol{W}}_{\mathrm{c}}^{-}$represent waves propagating in the positive and negative $z$ directions, respec-

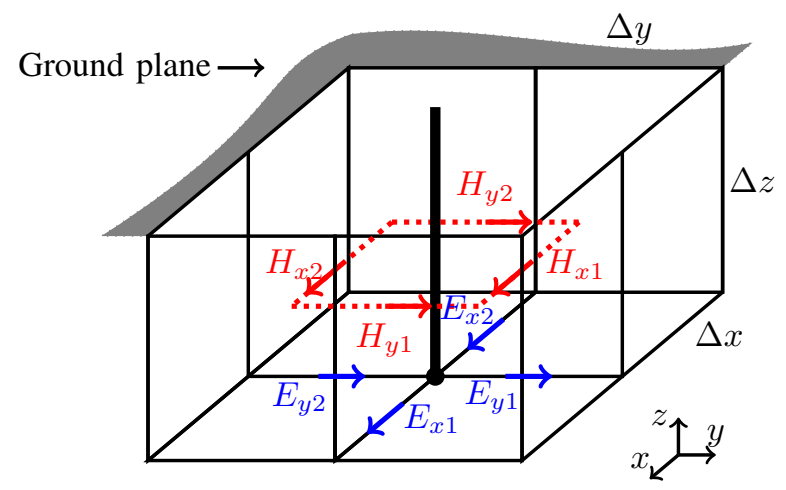

Fig. 16. Four Yee cells that model a $z$ directed discrete coaxial cable.

tively. Integrating over the annular surface $\Gamma_{\text {coax }}$ (Fig. 1), we find that

$$
\frac{1}{2 \pi \sqrt{\epsilon_{c}}} \int_{a}^{b} \int_{C_{r}} \boldsymbol{r} \cdot \tilde{\boldsymbol{W}}_{\mathrm{c}}^{ \pm} \frac{1}{r} d l d r=V \pm Z_{\mathrm{c}} I_{z}
$$

where $I_{z}$ is the current in the core (positive in the $z$-direction), $V$ is the potential difference between the inner and the outer conductor, and

$$
Z_{\mathrm{c}}=Z_{\mathrm{m}} \kappa, \quad Z_{\mathrm{m}}=\sqrt{\mu_{c} / \epsilon_{c}}, \quad \kappa=\frac{\log b / a}{2 \pi} .
$$

Expression (21) allows equation (19) to be rewritten in terms of the potential difference and the current as

$$
\frac{\partial}{\partial t}\left(V \pm Z_{\mathrm{c}} I_{z}\right) \pm c \frac{\partial}{\partial z}\left(V \pm Z_{\mathrm{c}} I_{z}\right)=0,
$$

with $V \pm Z_{\mathrm{c}} I_{z}$ represent signals propagating in the positive $(+)$ and negative $(-) z$ directions.

Consider four neighboring Yee cells whose upper faces reside on the same level as the ground plane (Fig. 16). To impose a TEM mode in these cells, the electric and magnetic field components in the $z$ direction, $E_{z}$ and $H_{z}$, should vanish. The value of $E_{z}$ can be set directly to zero by setting high conductivity values, $\sigma_{z}$, at the center and the outer perimeter of the four Yee cells. Moreover, applying Faraday's law

$$
\mu \int_{S} \frac{\partial H_{z}}{\partial t}=-\oint_{\partial S} \tilde{\boldsymbol{E}} \cdot d \boldsymbol{l}
$$

to each bottom face in Fig. 16, we see that $\partial H_{z} / \partial t$ and thus $H_{z}$ (since the initial condition is zero) can be made to vanish by requiring

$$
E_{x 1}^{n+1} \Delta=-E_{x 2}^{n+1} \Delta=E_{y 1}^{n+1} \Delta=-E_{y 2}^{n+1} \Delta \stackrel{\text { def }}{=} V^{n+1},
$$

where we have chosen $\Delta x=\Delta y=\Delta z=\Delta$ and defined $V^{n+1}$ as the discrete potential difference between the inner and the outer conductor of the cylindrical coaxial cable, located as illustrated in Fig. 17. Further, by a four point quadrature rule applied to Ampere's law, the current in the cylindrical coaxial cable evaluates to

$$
I_{z}^{n+\frac{1}{2}}=\frac{\pi \Delta}{4}\left(H_{y 1}^{n+\frac{1}{2}}-H_{y 2}^{n+\frac{1}{2}}+H_{x 2}^{n+\frac{1}{2}}-H_{x 1}^{n+\frac{1}{2}}\right) .
$$




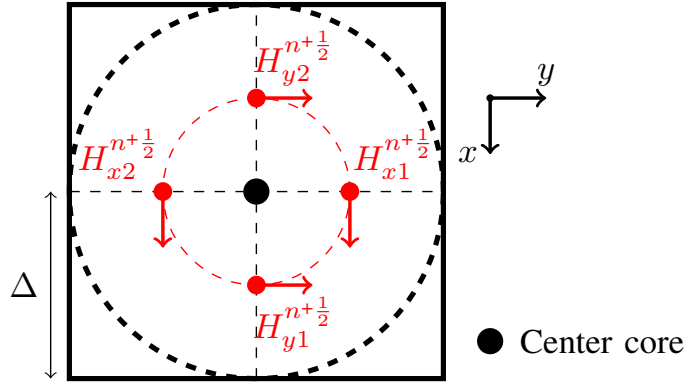

Fig. 17. A cross section through the cavity in Fig. 16, where for discrete impedance calculation, we assume a discrete cylindrical coaxial cable that has an outer shield (the thick dashed line) with radius equal to $\Delta$. is located as illustrated.

In the Yee grid, a discrete approximation of expression (20) yields

$$
\tilde{\boldsymbol{W}}_{\mathrm{c}}^{\Delta \pm}=\sqrt{\epsilon_{c}} \tilde{\boldsymbol{E}}^{n+1} \mp \sqrt{\mu_{c}} \boldsymbol{k} \times \tilde{\boldsymbol{H}}^{n+\frac{1}{2}}
$$

where the field values of Fig. 16 define $\tilde{\boldsymbol{W}}_{\mathrm{c}, 1}^{\Delta \pm}, \tilde{\boldsymbol{W}}_{\mathrm{c}, 2}^{\Delta \pm}, \tilde{\boldsymbol{W}}_{\mathrm{c}, 3}^{\Delta \pm}$, and $\tilde{\boldsymbol{W}}_{\mathrm{c}, 4}^{\Delta \pm}$, associated with the four inner vertical planes. Let $\boldsymbol{r}_{1}, \boldsymbol{r}_{2}, \boldsymbol{r}_{3}$, and $\boldsymbol{r}_{4}$ be unit vectors in the $x-y$ plane pointing from the center core towards the evaluation points of each $\tilde{\boldsymbol{W}}_{\mathrm{c}, i}^{\Delta \pm}$. Summing $\boldsymbol{r}_{i} \cdot \tilde{\boldsymbol{W}}_{\mathrm{c}, i}^{\Delta \pm}$ over the evaluation points, substituting equations (25) and (26), and rearranging the terms, yield

$$
\frac{\Delta}{4 \sqrt{\epsilon_{c}}} \sum_{i=1}^{4} \boldsymbol{r}_{i} \cdot \tilde{\boldsymbol{W}}_{\mathrm{c}, i}^{\Delta \pm}=V^{n+1} \pm \hat{Z}_{\mathrm{c}} I_{z}^{n+\frac{1}{2}}
$$

where $\hat{Z}_{c}=Z_{m} \hat{\kappa}$, with $\hat{\kappa}=1 / \pi$. Expression (28) constitutes a discrete version of expression (21). We note the different values of the geometric coefficients $\kappa$ and $\hat{\kappa}$.

\section{ACKNOWLEDGMENT}

This work is supported financially by the Swedish Research Council. The computations were performed on resources provided by the Swedish National Infrastructure for Computing (SNIC) at the High Performance Computing Center North (HPC2N). The authors thank Robin Augustine, Department of Engineering Sciences, Uppsala University, for help with the CST simulations.

\section{REFERENCES}

[1] C. A. Balanis, Modern Antenna Handbook. John Wiley \& Sons, 2008.

[2] M. N. Sadiku, Numerical Techniques in Electromagnetics, 2nd ed. CRC Press, 2001.

[3] L. Griffiths, C. Furse, and Y. C. Chung, "Broadband and multiband antenna design using the genetic algorithm to create amorphous shapes using ellipses," IEEE Trans. Antennas Propag., vol. 54, no. 10, pp. 2776 -2782 , Oct. 2006.

[4] N. Jin and Y. Rahmat-Samii, "Parallel particle swarm optimization and finite- difference time-domain (PSO/FDTD) algorithm for multiband and wide-band patch antenna designs," IEEE Trans. Antennas Propag., vol. 53, no. 11, pp. 3459 - 3468, Nov. 2005.

[5] J. Perez and J. Basterrechea, "Comparison of different heuristic optimization methods for near-field antenna measurements," IEEE Trans. Antennas Propag., vol. 55, no. 3, pp. 549 -555, Mar. 2007.

[6] S. Karimkashi and A. Kishk, "Invasive weed optimization and its features in electromagnetics," IEEE Trans. Antennas Propag., vol. 58, no. 4, pp. $1269-1278$, Apr. 2010.

[7] O. Sigmund, "On the usefulness of non-gradient approaches in topology optimization," Struct. Multidiscip. Optim., vol. 43, pp. 589-596, 2011.
[8] M. P. Bendsoe and O. Sigmund, Topology Optimization. Theory, Methods, and Applications. Springer, 2003.

[9] E. Wadbro, "Topology optimization for wave propagation problems," Ph.D. dissertation, Division of Scientific Computing, Uppsala Univ., Uppsala, Sweden, 2009.

[10] J. Jensen and O. Sigmund, "Topology optimization for nano-photonics," Laser Photon. Rev., vol. 5, no. 2, pp. 308-321, 2011.

[11] Y.-S. Chung, B.-J. Lee, and S.-C. Kim, "Optimal shape design of dielectric micro lens using FDTD and topology optimization," J. Opt. Soc. Korea, vol. 13, no. 2, pp. 286-293, Jun. 2009.

[12] G. Allaire, F. Jouve, and A.-M. Toader, "A level-set method for shape optimization," C. R. Acad. Sci. Paris Sér. I Math., vol. 334, no. 12, pp. 1125-1130, 2002.

[13] E. Wadbro and M. Berggren, "Megapixel topology optimization on a graphics processing unit," SIAM Review, vol. 51, no. 4, pp. 707-721, 2009.

[14] M. Gustafsson and S. He, "An optimization approach to twodimensional time domain electromagnetic inverse problems," Radio Science, vol. 35, no. 2, pp. 525-536, Mar. 2000.

[15] A. Bondeson, Y. Yang, and P. Weinerfelt, "Shape optimization for radar cross sections by a gradient method," Int. J. Num. Meth. Eng., vol. 61 no. 5, pp. 687-715, 2004.

[16] P. Jacobsson and T. Rylander, "Shape optimization of the total scattering cross section for cylindrical scatterers," Radio Science, vol. 44, no. 4, Aug. 2009.

[17] N. Nikolova, H. Tam, and M. Bakr, "Sensitivity analysis with the FDTD method on structured grids," IEEE Trans. Microw. Theory Tech., vol. 52 no. 4, pp. 1207 - 1216, Apr. 2004

[18] T. Nomura, K. Sato, K. Taguchi, T. Kashiwa, and S. Nishiwaki, "Structural topology optimization for the design of broadband dielectric resonator antennas using the finite difference time domain technique," Int. J. Num. Meth. Eng., vol. 71, pp. 1261-1296, 2007.

[19] Y.-S. Chung, C. Cheon, I.-H. Park, and S.-Y. Hahn, "Optimal design method for microwave device using time domain method and design sensitivity analysis. II. FDTD case," IEEE Trans. Magn., vol. 37, no. 5, pp. $3255-3259$, Sep. 2001

[20] E. Abenius and B. Strand, "Solving inverse electromagnetic problems using FDTD and gradient-based minimization," Int. J. Num. Meth. Eng., vol. 68, no. 6, pp. 650-673, 2006.

[21] D. Dyck, D. Lowther, and E. Freeman, "A method of computing the sensitivity of electromagnetic quantities to changes in materials and sources," IEEE Trans. Magn., vol. 30, no. 5, pp. 3415 -3418, Sep. 1994.

[22] D. Dyck and D. Lowther, "Automated design of magnetic devices by optimizing material distribution," IEEE Trans. Magn., vol. 32, no. 3, pp. 1188 -1193, May 1996.

[23] G. Kiziltas, D. Psychoudakis, J. Volakis, and N. Kikuchi, "Topology design optimization of dielectric substrates for bandwidth improvement of a patch antenna," IEEE Trans. Antennas Propag., vol. 51, no. 10, pp. 2732 - 2743, Oct. 2003.

[24] A. Erentok and O. Sigmund, "Topology optimization of sub-wavelength antennas," IEEE Trans. Antennas Propag., vol. 59, no. 1, pp. 58 -69, Jan. 2011.

[25] S. Zhou, W. Li, and Q. Li, "Level-set based topology optimization for electromagnetic dipole antenna design," J. Comput. Phys., vol. 229, no. 19 , pp. 6915 - 6930, 2010.

[26] A. Taflove and S. Hagness, Computational Electrodynamics: The FiniteDifference Time-Domain Method, 3rd ed. Artech House, 2005.

[27] M. Gustafsson and S. Nordebo, "Optimal antenna currents for Q, superdirectivity, and radiation patterns using convex optimization," IEEE Trans. Antennas Propag., vol. 61, no. 3, pp. 1109-1118, Mar. 2013.

[28] S. Gedney, "An anisotropic perfectly matched layer-absorbing medium for the truncation of FDTD lattices," IEEE Trans. Antennas Propag., vol. 44, no. 12, pp. 1630 -1639, Dec. 1996.

[29] K. Yee, "Numerical solution of initial boundary value problems involving maxwell's equations in isotropic media," IEEE Trans. Antennas Propag., vol. 14, no. 3, pp. 302 -307, May 1966.

[30] W. Gautschi, Numerical analysis: an introduction. Cambridge, MA, USA: Birkhauser Boston Inc., 1997.

[31] K. Svanberg, "A class of globally convergent optimization methods based on conservative convex separable approximations," SIAM J. Optim., vol. 12, no. 2, pp. 555-573, 2002.

[32] E. Hassan, E. Wadbro, and M. Berggren, "Topology optimization of UWB monopole antennas," in EUCAP 2013, Gothenburg, Sweden, Apr. 2013, pp. 1429-1433.

[33] A. Walther and A. Griewank, "Advantages of binomial checkpointing for memory-reduced adjoint calculations," in Numerical Mathematics and Advanced Applications. Springer, 2004, pp. 834-843. 
This is the author's version of an article that has been published in this journal. Changes were made to this version by the publisher prior to publication.

The final version of record is available at http://dx.doi.org/10.1109/TAP.2014.2309112

[34] N. Farahat and R. Mittra, "Analysis of frequency selective surfaces using the finite difference time domain (FDTD) method," in IEEE AP-S Int. Symp., vol. 2, 2002, pp. 568-571.

[35] E. C. Jordan and K. G. Balmain, Electromagnetic waves and radiating systems, 2nd ed. Englewood Cliffs, N.J. : Prentice-Hall, 1968.

[36] J. Maloney, G. Smith, and W. Scott, "Accurate computation of the radiation from simple antennas using the finite-difference time-domain method," IEEE Trans. Antennas Propag., vol. 38, no. 7, pp. 1059-1068, Jul. 1990.

[37] J. Maloney, K. Shlager, and G. Smith, "A simple FDTD model for transient excitation of antennas by transmission lines," IEEE Trans. Antennas Propag., vol. 42, no. 2, pp. 289 -292, Feb. 1994.

[38] S.-Y. Hyun, S.-Y. Kim, and Y.-S. Kim, "An equivalent feed model for the FDTD analysis of antennas driven through a ground plane by coaxial lines," IEEE Trans. Antennas Propag., vol. 57, no. 1, pp. 161-167, Jan. 2009.

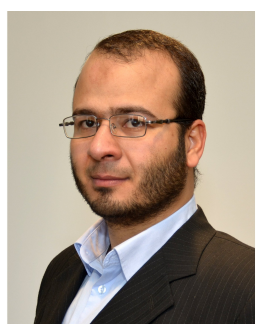

Emadeldeen Hassan received his B.S. and M.S. degree in electronics and communication engineering at Menofia University in 2001 and 2006, respectively. 2003-2010, he was a teaching assistant at the department of electronics and electrical communication engineering, Menofia University. Currently he is pursuing the Ph.D. degree at Umeå University.

His research interests include numerical methods in EM, EBG, defected ground structures, non-destructive evaluation and testing, optimization methods, and parallel programming.

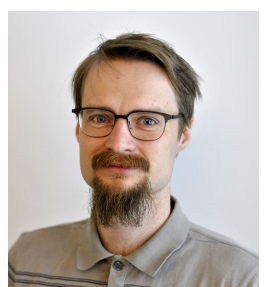

Eddie Wadbro received his M.Sc. degree in Mathematics at Lund University in 2004 and his Licentiate and Ph.D. degrees in Scientific Computing at Uppsala University in 2006 and 2009, respectively. Since 2009 , he works as an assistant professor at the Department of Computing Science, Umeå University.

His research interests concern mathematical modeling, development and analysis of efficient numerical methods for differential equations, primarily within the fields of design optimization and inverse problems.

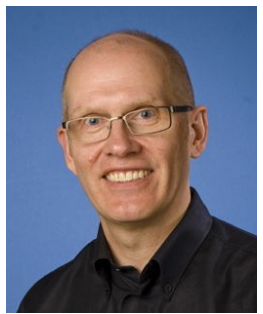

Martin Berggren is professor of Scientific Computing at Umeå University. He received his Ph.D. in Computational and Applied Mathematics at Rice University in 1996. Previously, he held a lectureship position at Uppsala University and research positions at FOI, the Swedish Defence Research Agency, and at Sandia National Laboratories, Albuquerque.

His research interests concern numerical methods for partial differential equations, and in particular computational design optimization for acoustic and electromagnetic wave propagation problems. 